MATEC Web of Conferences 16, 04002 (2014)

DOI: $10.1051 /$ matecconf/ 20141604002

C Owned by the authors, published by EDP Sciences, 2014

\title{
On the control of bistability in non-contact mode AFM using modulated time delay
}

\author{
I. Kirrou, M. Belhaq \\ Laboratory of Mechanics, University Hassan II-Casablanca, Morocco
}

\begin{abstract}
We study the control of bistability in non-contact mode AFM using time delay with modulated feedback gain. We consider that the tip-sample interaction force is described by Lennard-Jones potential and the equation of motion is modeled by single degree of freedom system. Perturbation analysis is performed to obtain the modulation equations of the slow dynamic. The influence of the modulated time delay on the nonlinear characteristic of the frequency response is analyzed and the evolution of the bistability region in the modulated time delay parameter plan is examined. Results show that modulation of the feedback gain can be used to reduce the amplitude of the microcanteliver response and to suppress the bistability regime in large region of the modulated delay parameter space. The analytical predictions are compared to numerical simulations for validation.
\end{abstract}

\section{Introduction}

Atomic Force Microscopy (AFM) is used to provide hightresolution surface topography at the atomic and molecular level. The dynamic of AFM basically depends on the interaction of a microcantilever with surface forces through a surface-tip interaction potential. This interaction property between the tip and the sample is exploited for material characterization and quantitative measurements of surface mechanical properties. One of the performance modes of AFM operation is the non-contact mode for which the probe operates in the attractive force region while remains separated from the sample without affecting it. In this context, the tip-sample interaction is minimized such that its dynamic is influenced by particular interaction forces. For instance, in non-contact mode the forces produced by the tip on the sample decrease so that inconveniences encountered in contact-mode are avoided. In most situations, such interactions cause the tip to oscillate vertically at a low amplitude with low (attractive) force offering advantages for characterization of soft samples [1]. Indeed, while repulsive interactions produce hardening effect to the response $[2,3]$ leading the tip oscillation to switch from a purely non-contact to tapping mode, attractive forces lead to softening behavior [4] causing bistability in the system (hysteresis) during frequency sweep. This bistable regime causes in general discontinuous transition of imaging characteristics which is a serious inconvenient when scanning soft matter. To avoid this undesirable phenomenon, it is necessary to develop strategies for controlling and suppressing the bistable regime.

The dynamic of a microcantilever-tip-sample interaction in non-contact mode has been studied by many authors, see for instance [5,6]. Ruetzel et al. [7] applied the Gakerkin method to truncate atomic force microscope subjected to tip-surface interaction described by the LennardJones potentials and calculated numerically periodic and chaotic behaviors.
On the other hand, several studies were conducted to control microcantilevers in dynamic force microscopy using time delayed feedback [8], or to eliminate chaotic motions in tapping mode AFM [9] or to control quality factor in dynamic force microscopy [10]. Experimental and theoretical studies were conducted by Alsaleem and Younis $[11,12]$ to analyze stabilization of capacitive MEMS resonator using a delayed velocity feedback controller. They showed that such a controller stabilizes MEMS resonators and enhances the stability near the pull-in band.

Using time delayed feedback to control the stability in AFM has also been considered in [13] demonstrating the experimental stabilization of cantilever oscillations. Motivated by this experimental investigation on controlling chaotic response in AFM using time delay, the present paper deals with the control of bistability in non-contact mode AFM near primary resonance using time delay. We assume that the system is under Lennard-Jones forces and we consider a lumped single degree of freedom model describing weakly nonlinear responses of the system. According to [13], the time-delayed feedback controller is introduced in velocity such that the control input is given by the error between the current output and the retarded one [14]. The main purpose here is the control of bistability using time delay with modulated feedback gain having a frequency higher than the naturel frequency and the frequency of the external excitation of the microcantilever.

The paper is organized as follows: Section 2 presents the equation of motion under Lennard-Jones forces in the presence of time-delayed feedback. The method of direct partition of motion (DPM) is applied to drive the main equation governing the slow dynamic of the system. In Section 3 we perform the method of multiple scales (MMS) on the slow dynamic to obtain the amplitude-phase modulation equations near primary resonance. Results of parameters effect on the frequency response are reported and discussed. Section 4 concludes the work. 


\section{Model and slow dynamic equation}

Considering the first mode of vibration in the AFM dynamic, the microcantilever tip motion can be modeled by a lumped single degree of freedom system, as shown in Fig. 1. If we assume that the microcantilever is subjected to a harmonic base excitation $d(t)=F \sin \left(\Omega_{1} t\right)$ and under time-delayed feedback, the equation of motion of the microcantilever tip can be represented in the following form

$$
\begin{array}{r}
m \ddot{x}+c_{0}(\dot{x}-\dot{d})+k_{0}(x-d)=f_{L J}+\left(G_{0}^{*}\right. \\
\left.+G_{1}^{*} \cos \Omega_{2} t\right)\left[\dot{x}\left(t-\tau_{d}^{*}\right)-\dot{x}(t)\right]
\end{array}
$$

where $x$ is the cantilever tip displacement relative to the

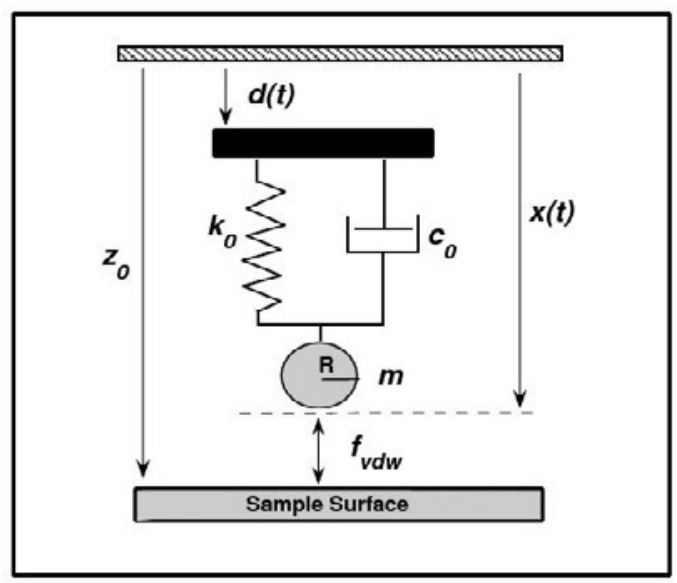

Fig. 1. The equivalent lumped-parameters model of the AFM microcantilever.

fixed base frame, $m, c_{0}$, and $k_{0}$ denote, respectively, the cantilever tip mass, damping and spring stiffness coefficients, and $f_{L J}$ denotes Lennard-Jones attraction/repulsion force (AFM tip-sample surface interaction force) for lumped parameters model defined explicitly by [7]

$$
f_{L J}=\frac{A_{2} R}{6\left(z_{0}-x\right)^{2}}-\frac{A_{1} R}{180\left(z_{0}-x\right)^{8}}
$$

where $z_{0}$ is the distance from the fixed base frame coordinate to the sample in the absence of any interaction between the tip and the sample, $A_{1}, A_{2}$ are the Hamaker constants for the repulsive and attractive potentials, respectively, and $R$ represents the cantilever tip radius. The coefficients $G_{0}^{*}, G_{1}^{*}$ are the unmodulated and the modulated gains, respectively, $\tau_{d}^{*}$ the time delay and $\Omega_{2}$ is the frequency of the modulated gain. The displacement $x$ is defined by considering the static problem as $x=z_{s}+X$ and the quantity $z^{*}=z_{0}-z_{s}$ as the equilibrium gap between the tip and the sample, where $z_{s}$ is the static position and $X$ is the displacement from the static position.

In order to facilitate the system identification, it is useful to normalize Eq. (1) using the following dimensionless variables: $u=\frac{X}{z^{*}}, \tau=\omega_{0} t, \tau_{d}=\omega_{0} \tau_{d}^{*}, \omega_{0}^{2}=\frac{k_{0}}{m}, \omega=\frac{\Omega_{1}}{\omega_{0}}$, $\Omega=\frac{\Omega_{2}}{\omega_{0}}, c=\frac{c_{0}}{m \omega_{0}}, G_{0}=\frac{G_{0}^{*}}{m \omega_{0}}, G_{1}=\frac{G_{1}^{*}}{m \omega_{0}}$ and $f=\frac{F}{z^{*}}$.

Expanding the Lennard-Jones force around the equilibrium position using the Taylor series expansion, the dimensionless equation of motion takes the form

$$
\begin{aligned}
\ddot{u}+\omega_{1}^{2} u+c \dot{u}+ & \beta_{2} u^{2}+\beta_{3} u^{3}=f \sin \omega \tau+c \omega f \cos \omega \tau \\
& +\left(G_{0}+G_{1} \cos \Omega \tau\right)\left[\dot{u}\left(\tau-\tau_{d}\right)-\dot{u}(\tau)\right](3)
\end{aligned}
$$

where $\omega_{1}^{2}=1+\beta_{1}, \beta_{1}=8 \alpha-2 \beta, \beta_{2}=36 \alpha-3 \beta, \beta_{3}=$ $120 \alpha-4 \beta$ with $\alpha=\frac{A_{1} R}{180 m \omega_{0}^{2} z^{* 9}}$ and $\beta=\frac{A_{2} R}{6 m \omega_{0}^{2} z^{* 3}}$.

Equation (3) includes a slow dynamic due to the excitation modelled as base harmonic motion and a fast dynamic produced by the frequency of the time-delayed feedback modulation $\Omega$ supposed larger than the natural and the external frequencies, $\omega_{1}$ and $\omega$, respectively.

To analyze the effect of the time-delayed feedback modulation on the frequency response of the slow dynamic (3), we use the method of DPM $[15,16]$. Averaging procedure gives the following equation governing the slow dynamic of the motion

$$
\begin{array}{r}
D_{1}^{2} z+c D_{1} z+\omega_{1}^{2} z+\beta_{2} z^{2}+\beta_{3} z^{3}-G_{0}\left[D_{1} z\left(T_{1}-\tau_{d}\right)\right. \\
\left.-D_{1} z\left(T_{1}\right)\right]+\left(\lambda_{1}+\lambda_{2} z\right)\left[D_{1}^{2} z+D_{1}^{2} z\left(T_{1}-\tau_{d}\right)\right. \\
\left.-2 D_{1} z\left(T_{1}\right) D_{1} z\left(T_{1}-\tau_{d}\right)\right]+\lambda_{3}\left[D_{1} z\left(T_{1}-2 \tau_{d}\right)\right. \\
\left.-D_{1} z\left(T_{1}-\tau_{d}\right)\right]-\lambda_{4} D_{1}^{2} z\left(T_{1}-\tau_{d}\right)+\lambda_{5} D_{1}^{2} z\left(T_{1}-2 \tau_{d}\right)
\end{array}
$$

$f \sin \omega \tau+c \omega f \cos \omega \tau(4)$

where $\lambda_{1}=\frac{\beta_{2} G_{1}^{2}}{2 \Omega^{4}}, \lambda_{2}=\frac{3 \beta_{3} G_{1}^{2}}{2 \Omega^{4}}, \lambda_{3}=\frac{G_{1}^{2}}{2 \Omega} \sin \Omega \tau_{d}, \lambda_{4}=\frac{G_{1}^{2}}{2 \Omega^{2}}+$ $\frac{G_{1}^{2}}{2 \Omega^{2}} \cos \Omega \tau_{d}$ and $\lambda_{5}=\frac{G_{1}^{2}}{2 \Omega^{2}} \cos \Omega \tau_{d}$.

\section{Frequency response analysis}

To study the dynamics of the microcantilever in the noncontact mode, we approximate the frequency-response curve of the slow dynamic near the primary resonance using the MMS $[17,18]$ and we examine the effect of modulated time-delayed feedback on the frequency response.

We perform the MMS to derive the modulation equations of amplitude and phase. Equilibria of these modulation equations corresponding to periodic response of the cantilever, are determined and analyzed.

The analysis of different regimes of the dynamic response of the tip-sample system is investigated for a representative case: the interaction of soft monocrystalline silicon microcantilever with the (111) reactive face of a flat silicon sample. The properties of the cantilever and interaction properties with respective samples are taken from [7], $k=0.11 \mathrm{~N} . \mathrm{m}^{-1}, R=150 \mathrm{~nm}, \omega_{0}=74129.12 \mathrm{rad} . \mathrm{s}^{-1}$, $Q=100, A_{1}=1.3596 \times 10^{-70} \mathrm{Jm}^{6}, A_{2}=1.865 \times 10^{-19} \mathrm{~J}$, and $f=0.008$.

Figure 2 shows the amplitude-frequency response in the absence of the feedback gains $\left(G_{0}=0\right.$ and $\left.G_{1}=0\right)$ for decreasing the gap between the tip and the sample $z^{*}$. The solid lines correspond to stable branches while the dashed line corresponds to the unstable one. The plots depict that as the gap between the tip and the sample is increased, the softening nonlinear response shifts toward higher frequencies.

Next we study the effect of time delay on the dynamic of non-contact mode AFM near the primary resonance in the case where the time delay has unmodulated gain only $\left(G_{0} \neq 0, G_{1}=0\right)$. We chose the parameters $c=0.01, z^{*}=$ $7 \mathrm{~nm}$ and we analyze the frequency response for different values of $G_{0}$ and $\tau_{d}$.

Figure 3 illustrates the frequency response versus external frequency $\omega$ for different values of the gain $G_{0}$. Analytical approximations (solid (dashed) lines correspond to stable (unstable) solutions) are compared to numerical 

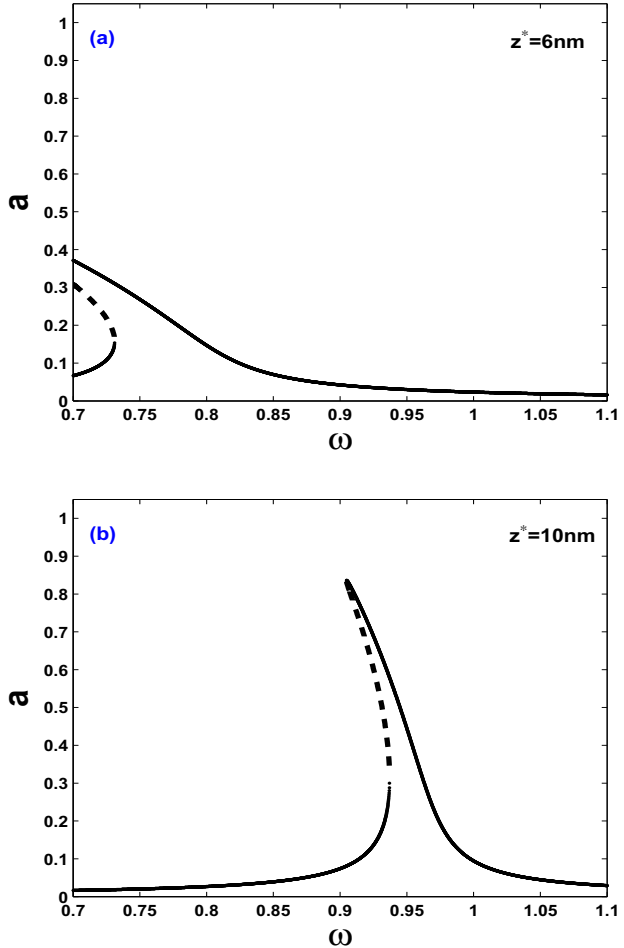

Fig. 2. Frequency response for $G_{0}=0, G_{1}=0$ and for different values of the gap $z^{*}$; (a) $z^{*}=6 \mathrm{~nm}$, (b) $z^{*}=10 \mathrm{~nm}$.
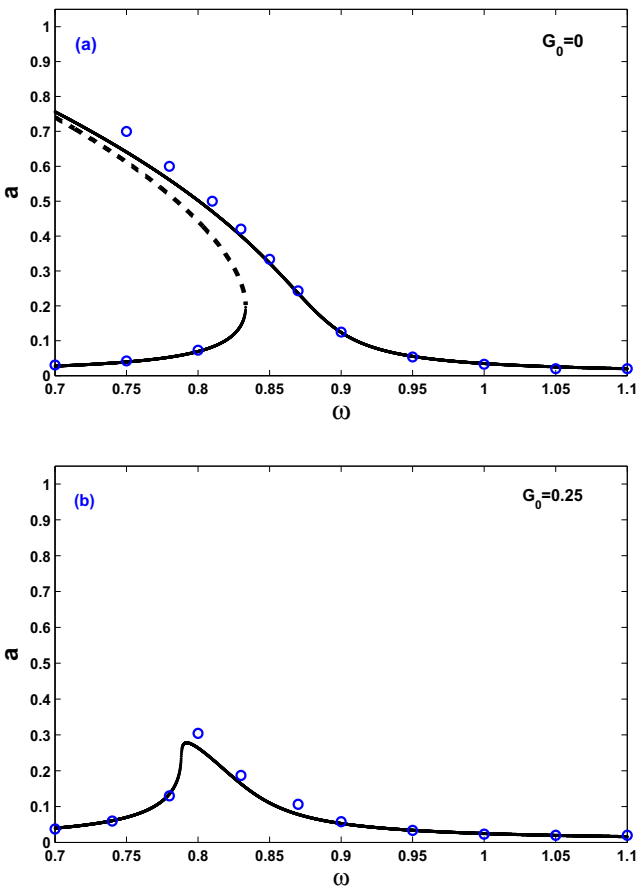

Fig. 3. Frequency response for $\Omega=4, \tau_{d}=0.5$ and $G_{1}=0$; (a) $G_{0}=0$, (b) $G_{0}=0.25$. Solid lines: analytical approximation; circle: numerical simulation.

simulations (circles). It can be seen that increasing the gain $G_{0}$ causes a decrease in the amplitude and suppression of bistability.

The effect of the modulated feedback gain $G_{1}$ on the dynamic of non-contact mode AFM near the primary resonance is shown in Fig. 4 for a given value of $G_{0}$ and $\tau_{d}$.
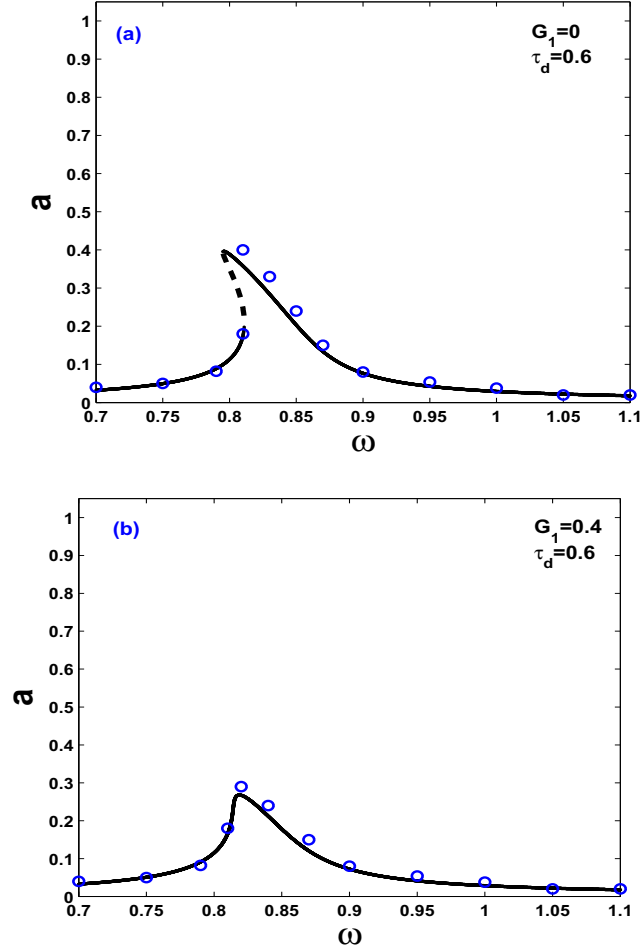

Fig. 4. Frequency response for $G_{0}=0.1, \tau_{d}=0.6$ and for different values of the gain $G_{1}$

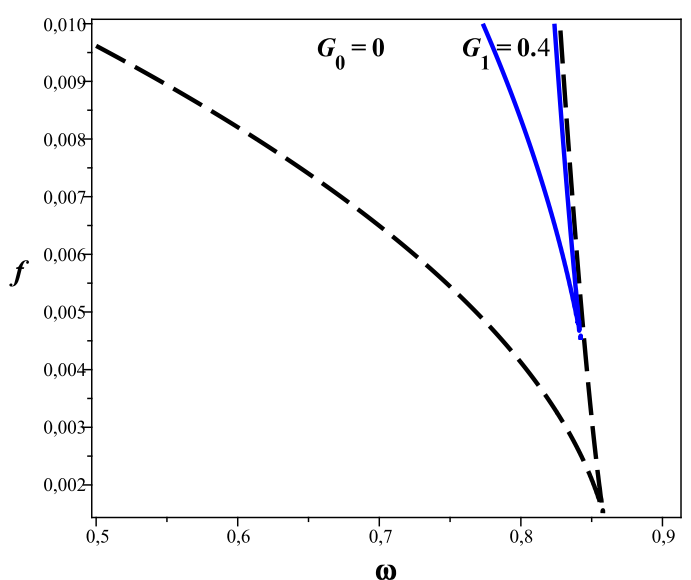

Fig. 5. Bifurcation curves of periodic solutions in the plane $(f, \omega)$ for $f=0.008, \Omega=4$ and $G_{0}=0$.

It can be seen that $G_{1}$ also contributes to a decrease in the amplitude and to the bistability suppression.

To determine the bifurcation curves delimiting the region where bistability occurs, we examine the roots of the amplitude-frequency response equation corresponding to periodic solutions of Eq. (4). This equation has one or three real solutions depending on the sign of its discriminant $\Delta$. The loci of the bifurcation curves separating the existence domain of the stationary solutions are given by the condition $\Delta=0$ and are plotted in Fig. 5. The plot shows the bifurcation boundaries in the parameter plane $(f, \omega)$ for two different values of $G_{1}\left(G_{1}=0\right.$ for dashed lines and $G_{1}=0.4$ for solid lines). In the regions between the boundaries $(\Delta<0)$ three solutions exist, two stable and one unstable, while only one stable solution exists outside the 
boundaries $(\Delta>0)$. The stability analysis has been done using the Jacobian of the slow flow system. It can be seen from this figure that as the amplitude $G_{1}$ is increased, the domain of the bistability decreases significanly.

\section{Conclusions}

The effect of unmodulated and modulated gains on the frequency response of a non-contact mode AFM was studied in this work. A lumped single degree of freedom system modelling the cantilever dynamics of non-contact mode AFM was considered and emphasis was placed on the case when the AFM is driven near its primary resonance. The interaction force was assumed to be described by the Lennard-Jones model and the external harmonic force was derived from the base excitation of the microcantilever. The technique of direct separation of motion as well as the multiple scales technique were used to determine the nonlinear frequency response of the slow dynamic near the fundamental resonance.

The main results of this work is that the bistability regime in non-contact mode AFM can be controlled by the modulated and the unmodulated feedback gains. This result may contribute to tune the amplitude of the tip response at a stable required level in order to enhance the performance in the scanning operation in term of measuring forces involved in the operation.

\section{References}

[1] J. Kupnizky, Nanostructures studied by AFM, Thesis from Acta University (2003).

[2] B. Anczykowski, D. Krüger, H. Fuchs, Cantilever dynamics in quasinon-contact force microscopy: Spectroscopic aspects, Phys. Rev. B, 53, 1996, 485-488.

[3] A. Kühle, A.H. Soerensen, J. Bohr, Role of attractive forces in tapping tip force microscopy, J. Appl. Phys. 81, 1997, 6562-6569.

[4] R.W. Stark, Bistability, higher harmonics, and chaos in AFM, Materials Today, 13, 2010, 24-32.

[5] M. Tsukada, N. Sasaki, R. Yamura, N. Sato, K. Abe, Features of cantilever motion in dynamic-mode AFM, Surf. Sci. 401, 1998, 355-363.

[6] M. Lee, W. Jhe, General theory of amplitudemodulation atomic force microscopy, Phys. Rev. Lett, 97, 2006, id. 036104.

[7] S. Rützel, S.I. Lee, A. Raman, Nonlinear dynamics of atomic-force microscope probes driven in LennardJones potentials, Proc. R. Soc. Lond, 459, 2003, 19251948.

[8] K. Yamasue, T. Hikihara, Contol of microcantilevers in dynamic force microscopy using time delayed feedback, Rev. Sci. Instrum, 77, 2006, 1-6.

[9] H. Sadeghian, M.T. Arjmand, H. Salarieh, A. Alasty, Chaos control in single mode approximation of $\mathrm{T}$ AFM systems using nonlinear delayed feedback based on sliding mode control. In: Proceedings of the ASME 2007 International Design and Engineering Tchenical Conference and Computers and Information in Engineering Conference, Las Vegas, NV (2007).
[10] R.W. Stark, Time delay Q-control of the microcantilever in dynamic atomic force microscopy. In: Proceedings of 2005 5th IEEE Conference on Nanotechnology, Nagoya, Japan, (2005).

[11] F.M. Alsaleem and M.I. Younis, Integrity Analysis of Electrically Actuated Resonators With Delayed Feedback Controller, Journal of Dynamic Systems, Measurement, and Control, 133, 2013, 031011.

[12] F.M. Alsaleem and M.I. Younis, Stabilization of electrostatic MEMS resonators using a delayed feedback controller, Smart Materials and Structures, 19, 2010, 035016.

[13] K. Yamasue, K. Kobayashib, H. Yamada, K. Matsushige, T. Hikihara, Controlling chaos in dynamicmode atomic force microscope, Phys. Lett. A, 373, 2009, 3140.

[14] K. Pyragas, A. Tamaoevièius, Continuous control of chaos by self-controlling feedback, Phys. Lett. A, 170, 1992, 421-428.

[15] I.I. Blekhman, Vibrational Mechanics-Nonlinear Dynamic Effects, General Approach, Application. Singapore: World Scientific (2000).

[16] J.J. Thomsen, Vibrations and Stability: Advanced Theory, Analysis, and Tools. Springer-Verlag, BerlinHeidelberg (2003).

[17] A.H. Nayfeh, D.T. Mook, Nonlinear Oscillations. New York: Wiley (1979).

[18] A.H. Nayfeh, Introduction to Perturbation Techniques. New York: Wiley (1981). 